\title{
1 Eye contact marks the rise and fall of shared attention in \\ 2 conversation
}

4 Sophie Wohltjen ${ }^{1 *} \&$ Thalia Wheatley ${ }^{1,2}$

5 'Psychological and Brain Sciences Department, Dartmouth College, 6207 Moore Hall, Hanover, $6 \mathrm{NH}$ 03755; ${ }^{2}$ Santa Fe Institute, Santa Fe, NM

$7 \quad{ }^{*}$ Corresponding author: Sophie Wohltjen

8 Email: Sophie.E.Wohltjen.GR@dartmouth.edu

9 Author Contributions: SW and TW conceptualized and designed the experiment; SW collected 10 and analyzed the data; SW and TW wrote the manuscript.

11 Competing Interest Statement: The authors declare no competing interests.

12 Classification: Social Sciences, Psychological and Cognitive Sciences

13 Keywords: pupillometry, synchrony, eye contact, conversation, shared attention

14 This Word document includes:

$15 \quad$ Main Text

16

17

18

19

20

21

22

23

24 
26 Conversation is the platform where minds meet -the venue where information is shared, ideas co27 created, cultural norms shaped, and social bonds forged. Its frequency and ease belie its 28 complexity. Every conversation weaves a unique shared narrative from the contributions of 29 independent minds, requiring partners to flexibly move into and out of alignment as needed for 30 conversation to both cohere and evolve. How two minds achieve this coordination is poorly 31 understood. Here we test whether eye contact, a common feature of conversation, predicts this 32 coordination by measuring dyadic pupillary synchrony (a corollary of shared attention) during 33 natural conversation. We find that eye contact is positively correlated with synchrony as well as 34 ratings of engagement by conversation partners. However, rather than elicit synchrony, eye contact 35 commences as synchrony peaks and predicts its immediate and subsequent decline until eye 36 contact breaks. This relationship suggests that eye contact signals when shared attention is high.

37 Further, we speculate that eye contact may play a corrective role in disrupting shared attention 38 (reducing synchrony) as needed to facilitate independent contributions to conversation.

\section{Significance Statement}

41 Conversation is the platform where minds meet to create and exchange ideas, hone norms and 42 forge bonds. But how do minds coordinate with each other to build a shared narrative from 43 independent contributions? Here we show that when two people converse, their pupils periodically 44 synchronize, marking moments of shared attention. As synchrony peaks, eye contact occurs and 45 synchrony declines, only to recover as eye contact breaks. These findings suggest that eye contact 46 may be a key mechanism for enabling the coordination of shared and independent modes of 47 thought, allowing conversation to both cohere and evolve. 

Introduction

51 Good conversations proceed effortlessly, as if conversation partners share a single mind. This 52 effortlessness obscures the complexities involved. Conversation partners must weave shared 53 understanding from alternating independent contributions (Frith \& Frith, 2010; Heldner \& Edlund, 54 2010; Levinson \& Torreira, 2015; Stolk et al., 2016) in an act of spontaneous, dynamic co-creation.

55 Given too few independent insights, conversation stagnates. When there is insufficient common 56 ground, people talk past each other. Even deciding when conversation should end is a feat of social 57 coordination (Mastroianni, et al., 2021). Engaging conversation must continuously negotiate the 58 delicate balance between creating shared understanding while allowing the conversation to move 59 forward and evolve. How do two minds negotiate this balance?

61 There are several reasons to believe that eye contact may play an instrumental role. Eye contact62 when two people look at each other's eyes_occurs ubiquitously in conversation, often at the ends 63 of turns when partners pass the conversational baton (Mortensen, 2011; Hedge, et al., 1978; 64 Hirvenkari et al., 2013; Kendon, 1967). It is possible that the known effects of eye contact on arousal 65 (Binetti et al., 2016; Jarick \& Bencic, 2019; Mazur et al., 1980) and attention (Abney, et al., 2020; 66 Conty et al., 2006; Senju \& Hasegawa, 2005) may nudge partners at critical moments in the 67 conversation that facilitate this exchange. The ubiquity yet brevity of eye contact in natural 68 conversation, averaging 1.9s, (Kendon, 1967), also suggests that these attentional nudges are not 69 scattered randomly but occur at precise times to optimize the attention of both parties. Here we 70 tested whether eye contact has a particular relationship with shared attention during natural 71 conversation.

73 To quantify the dynamic wax and wane of shared attention during natural conversation, we 74 employed dyadic high temporal resolution pupillometry. Under light constancy, pupil diameter 
75 fluctuates coincident with neuronal activity in the locus coeruleus (Aston-Jones, et al., 1994;

76 Rajkowski, 1993), which is associated with attention (Alnaes, et al., 2014; Gilzenrat, et al., 2010;

77 Joshi et al., 2016). Further, an attention-inducing stimulus evokes an associated pupillary response

78 (i.e. a dilation and return to baseline -- Hoeks \& Levelt, 1993). Monitoring these pupil responses

79 over time provides a continuous index of attention (Kang et al., 2014; Kang \& Banaji, 2020; Van

80 den Brink, et al., 2016; Smallwood, et al., 2011; Wierda, et al., 2012). When people attend to the

81 same dynamic stimulus, their pupillary timeseries can be compared as a measure of shared

82 attention, with similar pupillary timeseries indexing similar timeseries of attention. Unlike joint

83 attention, shared attention does not require eye contact or gestures followed by attention to a third

84 object. Rather, shared attention can refer to any situation in which individuals are attending to the

85 same stimulus and can occur with or without eye contact (Shteynberg, 2015). While pupil mimicry

86 has been a documented outcome of looking at another's eyes (Prochazkova, et al., 2018; van

87 Breen, et al., 2018), shared attention can also exist in the absence of any visual stimulus (e.g., two

88 people listening to the same music - Kang et al., 2015).

90 We use the term "pupillary synchrony" to refer to how closely two conversation partners' pupillary

91 timeseries co-vary over time (Kang \& Wheatley, 2017). Previous studies have also used this

92 definition of synchrony to measure co-variation of other physiological and/or behavioral responses

93 (e.g., brain blood oxygenation levels- Hasson \& Frith, 2016; neuroelectrical activity- Leong, et al.,

94 2017, Dikker, et al., 2017, Kinreich, et al., 2017; heart-rate variability - Konvalinka, et al., 2011).

95 Here we used pupillary synchrony between conversation partners to capture temporal variation in

96 shared attention over the course of a natural conversation.

97

98 We are not the first to examine inter-subject synchrony and eye contact. Leong and colleagues

99 (2017) showed that infant-adult dyads exhibit more brain-to-brain synchrony in EEG while making 100 direct gaze than when interacting with their gaze averted. Similarly, students in real classrooms 
showed more neural synchrony in EEG while learning with classmates with whom they had previously made eye contact (Dikker et al., 2017). Kinreich et al. (2017) used EEG to measure synchrony while dyads planned a fun day to spend together and found that moments of eye contact were correlated with neural synchrony. Additionally, research with both fNIRS and hyperscanning fMRI paradigms have also found neural synchrony during eye contact in a wide variety of scenarios and across multiple brain regions (Hirsch et al., 2017; Kelley, et al., 2020; Koike et al., 2016; 2019; Noah et al., 2020), leading to the prevailing inference that eye contact enhances synchrony (Hirsch et al., 2017). Yet exactly how eye contact and synchrony are linked is not understood. Does eye contact precede synchrony, as enhancement would suggest, or follow it? Here we probe how eye contact is linked to pupillary synchrony by investigating the temporal relationship between the two.

It is also unclear when synchrony in conversation is desirable. The majority of the synchrony literature relies on tasks in which shared attention equates to better performance. For example, when individuals hear the same story or watch the same movie, the degree to which they have similar responses positively predicts their comprehension of (Silbert et al., 2014; Stephens et al., 2010) and shared memory for (Chen et al., 2017) the shared stimulus. But conversation involves more than perceiving a shared stimulus: it requires fluidly shifting between shared and independent modes of thought, from comprehension of what is being said to formulating one's next contribution (Bögels et al., 2015). These independent contributions allow a conversation to move and evolve. We therefore not only test the relationship between eye contact and pupillary synchrony, but also how both relate to success in conversation as measured by ratings of engagement (see Redcay \& Schilbach, 2019; Schilbach et al., 2013) made by the partners themselves.

In the present study, dyads engaged in unstructured conversation while their eyes were tracked. Afterward, they separately re-watched their conversations while continuously rating how engaged they remembered feeling. We show that eye contact is correlated with dyadic pupillary synchrony 
during these unstructured conversations, consistent with the research summarized above. Further, we find that eye contact has a distinct temporal relationship with pupillary synchrony consistent with

129 (though not proof of) a coordinative role. Conversation partners make eye-contact as pupillary 130 synchrony peaks, which may provide a communicative signal that shared attention is high. Pupillary 131 synchrony then immediately and sharply decreases until eye contact stops. This temporal 132 relationship suggests that eye contact (or its correlates) may actually reduce shared attention, 133 perhaps to allow for individual contributions that enable a conversation to evolve. Consistent with 134 this inference, we find that the amount of eye contact - not synchrony - predicts self-reported 135 engagement by conversation partners. Rather than maximizing shared attention, a good 136 conversation may require shifts into and out of a shared attentional state, with these shifts 137 accompanied by eye contact.

139 Results

140 Dyads are more synchronous during eye contact

141 A generalized linear mixed effects analysis was performed in $\mathrm{R}$ using the Ime4 package (Bates et 142 al., 2014) to investigate the relationship between dyads' pupillary synchrony and eye contact during 143 their conversations. Pupillary synchrony was computed in one-second windows using Dynamic 144 Time Warping (DTW), a well-established algorithm that allows and accounts for small offsets in 145 time between otherwise similar signals (see methods for a full description of this algorithm). For 146 this analysis, DTW scores were log-transformed to correct for homoscedasticity, and dyads' time 147 series of eye contact were down-sampled to $1 \mathrm{hz}$. The model included a fixed effect for pupillary 148 synchrony, random intercepts for dyads, and assumed a binomial distribution in order to determine 149 whether dyads were more synchronous during moments of eye contact vs. no eye contact.

151 There was a significant effect of pupillary synchrony on eye contact (log odds $=0.066$, odds ratio $152=1.07, \mathrm{Cl}=1.03-1.11, \mathrm{p}<0.001)$, demonstrating that dyads were more synchronous during eye 
contact than in the absence of eye contact. To more robustly test the probability that our effect of pupillary synchrony on eye contact was not due to chance, we compared our true effect estimate to 1) a null distribution created by shuffling where moments of eye contact occurred within a dyad's time series, in order to determine whether our true estimate was the result of random variation in eye contact, and 2) a null distribution created by shuffling intact eye contact time series between subjects, in order to see whether our true estimate was the result of common variation in eye contact across all conversations. Our true effect was significantly positively skewed from both of these distributions (see figure 1), suggesting that not only are pupillary synchrony and eye contact significantly related to one another, but they are also related to one another in ways that are unique

162 to the structure of individual dyadic conversations.

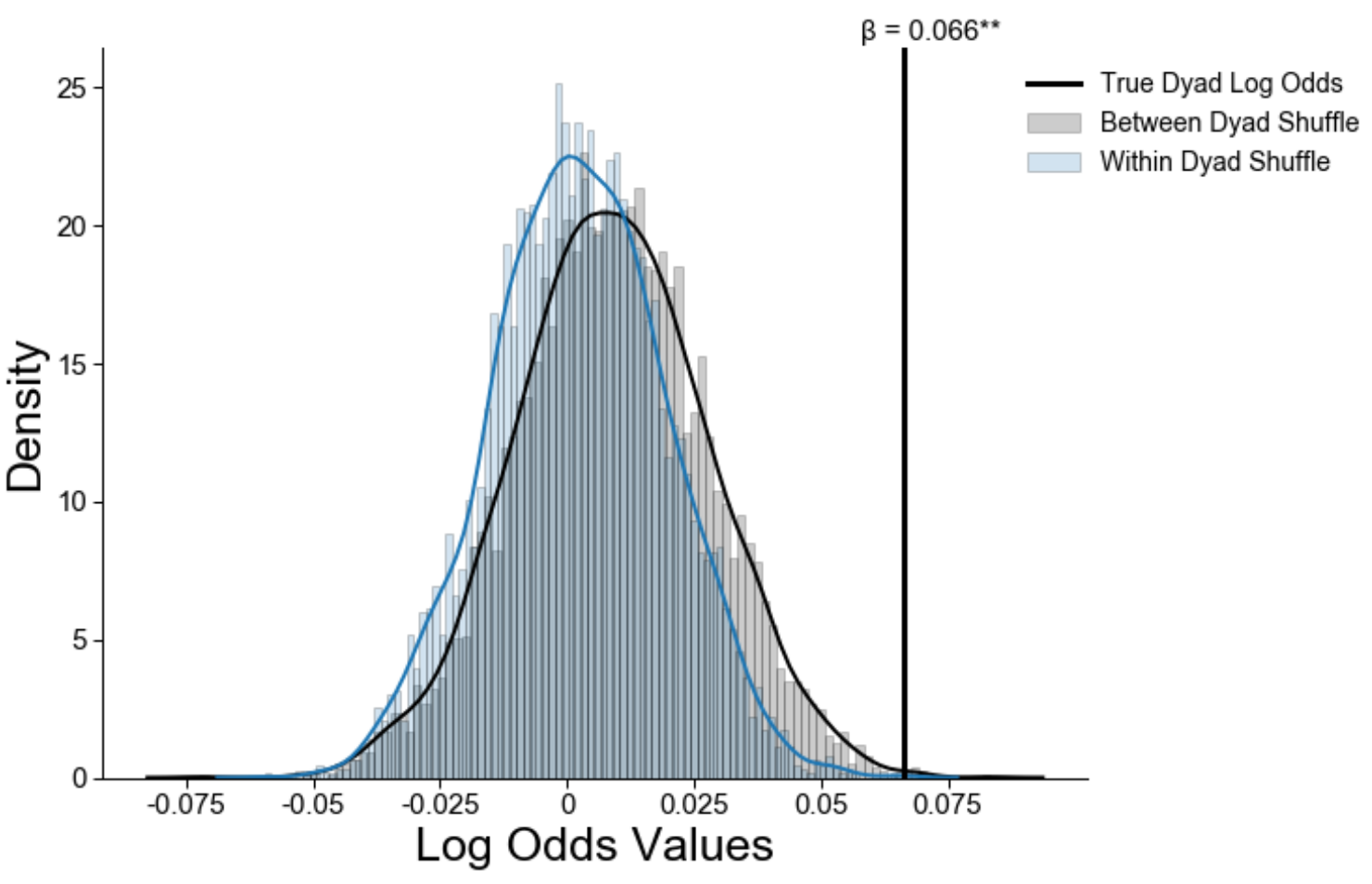

Figure 1. Results of a permutation test comparing the true log odds $(\beta=0.066)$ of the association 166 between eye contact and pupillary synchrony to 1) 5000 permutations shuffling dyads eye contact and pupillary synchrony time series and 2) 5000 permutations shuffling eye contact time series 
within each dyad. The true relationship between pupillary synchrony and eye contact fell significantly outside these two distributions.

\section{Eye contact follows (rather than elicits) synchrony}

\section{Multi-level Vector Auto-regression}

We investigated the directionality of the relationship between eye contact, pupil size, and pupillary synchrony using a Multi-level Vector Auto-regression analysis (mIVAR; see methods for full description). mIVAR outputs temporal, contemporaneous, and between-subjects networks to represent the directed and undirected relationships between variables of interest. Because mIVAR was designed for continuous time series, we parsed each binary time series of eye contact, comprised of samples where eye contact either was or was not being made, into one-second epochs and took the average of the eye contact in these epochs, resulting in a proportion of eye contact made per one-second window.

We found a significant between-subjects relationship for eye contact and pupillary synchrony (partial $r=0.25, p=0.01$ ), suggesting that dyads who made significantly more eye contact overall also showed higher levels of pupillary synchrony over their entire conversations. We also found contemporaneous network relationships between pupil size and eye contact (partial $r=0.02, p=$ 0.001 ) and pupil size and synchrony (partial $r=0.23, p<0.001$ ), suggesting that in moments when an individual's pupils dilate, they both make more eye contact and are more synchronous with their conversation partner. Finally, we found temporal network relationships suggesting that both pupil size (partial $r=0.04, p<0.001$ ) and synchrony (partial $r=0.03, p<0.001$ ) in one moment lead to eye contact in the next (all results shown in figure 2). While these $R$ values are small, this analysis rules out eye contact as a causal mechanism for creating pupillary synchrony. Instead, this temporal network result suggests that rather than being a strategy used to increase pupillary 
A

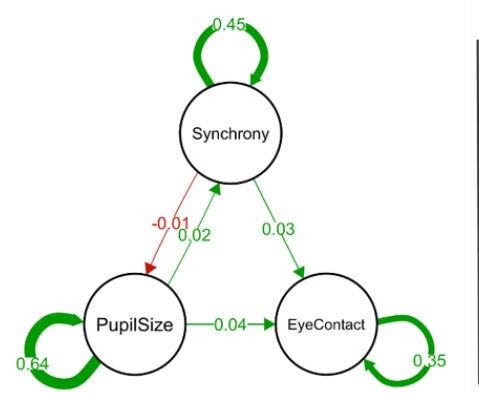

Temporal Network
B

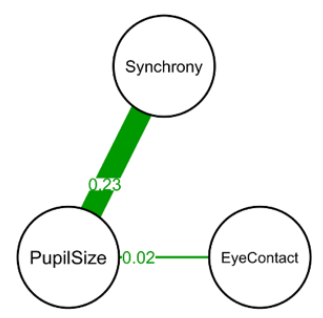

Contemporaneous Network
C

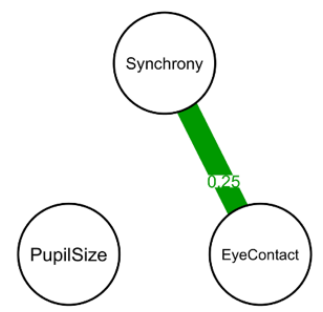

Between-Subjects Network

Figure 2. Gaussian graphical networks depicting the relationships between pupillary synchrony, pupil size, and eye contact. All edges depict significant relationships at $\alpha=0.05$. Edge thickness represents the size of each relationship, and specific edge weights are overlaid on top of each edge. Green edges represent positive relationships, and red edges represent negative relationships. A) Temporal network illustrating the relationship between pupillary synchrony, pupil size, and eye contact at one lag. Pupillary synchrony and pupil size temporally precede eye contact. B) Contemporaneous network illustrating the simultaneous relationships between pupillary synchrony, pupil size, and eye contact. Pupil size is positively related to both eye contact and to pupillary synchrony. C) Between subjects network illustrating the per-subject average relationship between pupillary synchrony, eye contact, and pupil size. Subjects who shared more eye contact during their conversations were more synchronous.

\section{Event-Related Analysis}

To further examine the temporal relationship between eye contact and pupillary synchrony, we investigated how pupillary synchrony fluctuates, on average, around a single instance of eye 
212 contact (see methods for full description of this analysis). For example, a dyad could synchronize

213 prior to the instance of eye contact and remain highly aligned for the duration, suggesting that

214 dyadic pupillary synchrony is maintained during eye contact. Conversely, a dyad could synchronize

215 prior to the onset of eye contact, but begin to move out of alignment during the instance, which

216 would suggest that pupillary synchrony is broken during moments of eye contact.

218 We found that pupillary synchrony begins to increase prior to the start of eye contact, peaks at the 219 onset of eye contact, and decreases immediately thereafter (figure 3).
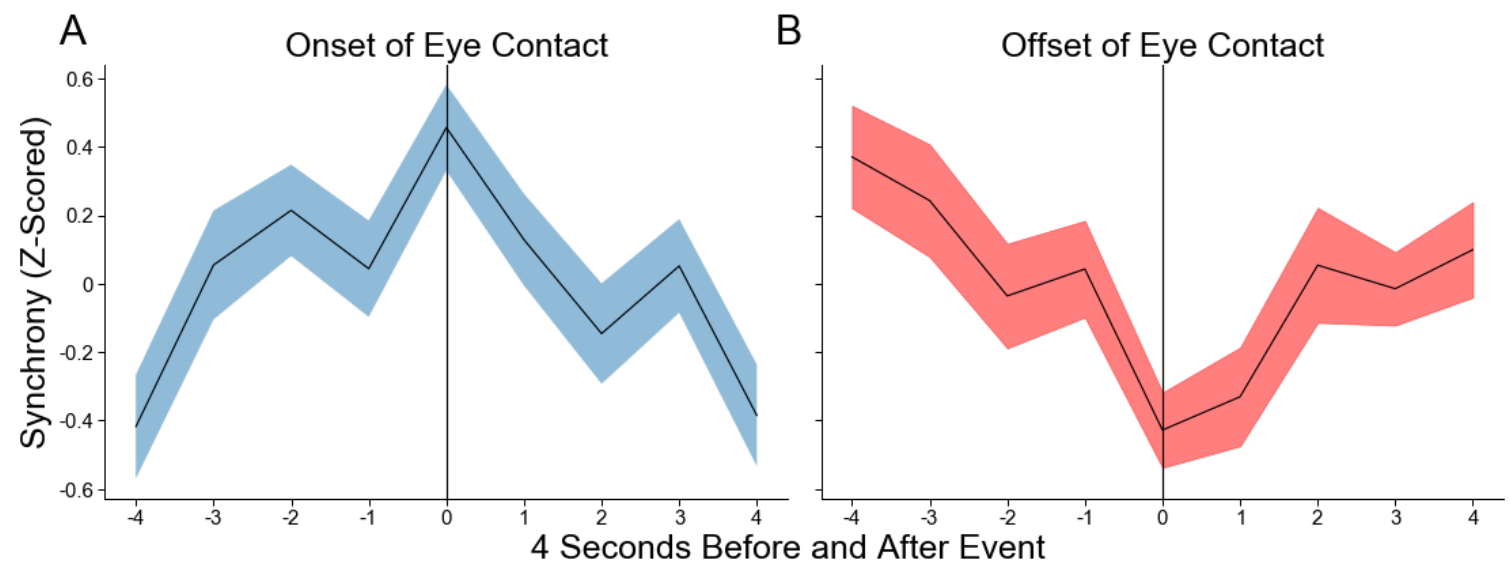

C

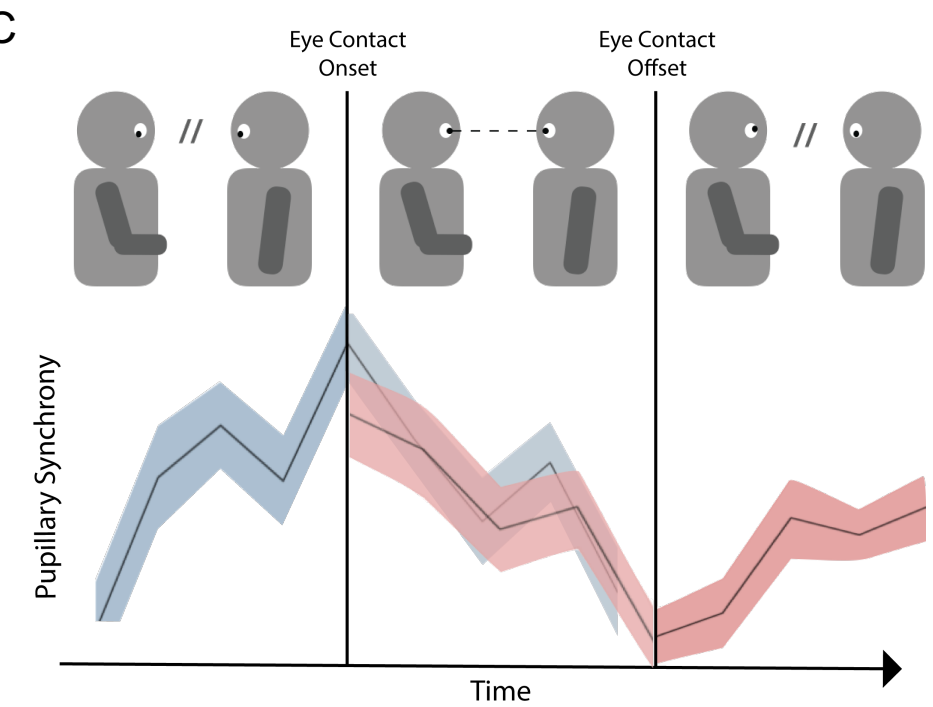


Figure 3. Time series of pupillary synchrony in the four seconds leading up to and following the A) onset of eye contact with standard error and B) offset of eye contact with standard error (mean duration of eye contact $=1.07 \mathrm{~s}, \mathrm{SD}=1.14)$. Peak and trough pupillary synchrony occur at the onset and offset of eye contact, respectively. C) Illustrative cartoon of how a single instance of eye contact coincides with pupillary synchrony. Prior to eye contact, pupillary synchrony increases until it peaks at eye contact onset (real data from panel A). As eye contact is maintained, synchrony declines until its trough when eye contact is broken (real data from panel B).

We statistically tested this effect by computing a linear model predicting the average pupillary synchrony curve per dyad and specifying planned contrasts for how synchrony might vary over time (e.g., linear, quadratic, cubic shapes). This model was significant $\left(f(8,414)=3.84, R^{2}=0.05\right.$, $p<0.001$ ), and results confirmed that a quadratic contrast was the best fit for dyadic pupillary synchrony $(\beta=-0.65, \mathrm{Cl}=-0.93--0.37, \mathrm{p}<0.001)$. These effects also proved to be robust to naturalistic data issues (e.g., irregular spacing and duration of eye contact; see supplementary information).

We also performed the same event-related analysis of pupillary synchrony surrounding the offset of eye contact-that is, when dyads broke eye contact. Here we found the opposite effect such that pupillary synchrony decreased leading up to the offset, reached its lowest point at the offset, then began to increase following the offset. We statistically tested this effect as well by computing a

242 linear model predicting our average pupillary synchrony curve per dyad and again specifying 243 planned contrasts for how pupillary synchrony might vary over time. This model was also significant $244\left(f(8,414)=3.08, R^{2}=0.04, p=0.002\right)$, and a quadratic contrast was also a significant fit for dyads' 245 pupillary synchrony curves surrounding the offset of eye contact $(\beta=0.53, \mathrm{Cl}=0.25-0.81, p<$ 246 0.001). These results suggest that although eye contact is positively associated with pupillary synchrony in general, it does not causally increase pupillary synchrony. Rather, eye contact occurs 
as pupillary synchrony peaks and persists over its decline until pupillary synchrony reaches a nadir. Pupillary synchrony only begins to increase again once the moment of eye contact ends.

To further test whether this parabolic contour was peculiar to moments surrounding eye contact, we compared true eye contact onsets and offsets to a randomly sampled, equal number of moments in the conversation where eye contact was not made, creating "pseudo onsets" and 254 "pseudo offsets." We calculated dyadic pupillary synchrony curves for the four seconds prior and 255 four seconds following these randomly chosen conversation points, repeating the process 1000 256 times for each dyad in order to compare the distribution of pupillary synchrony at pseudo onsets and offsets to pupillary synchrony at true eye contact onsets and offsets. For both onsets and offsets, the true pupillary synchrony value fell outside the null distribution (see figure 4). Thus, eye contact onsets and offsets are associated with more pupillary synchrony $(Z=0.46, p<0.001)$ and less pupillary synchrony $(Z=-0.43, p<0.001)$, respectively, than what could be expected from natural fluctuations of pupillary synchrony during conversation.

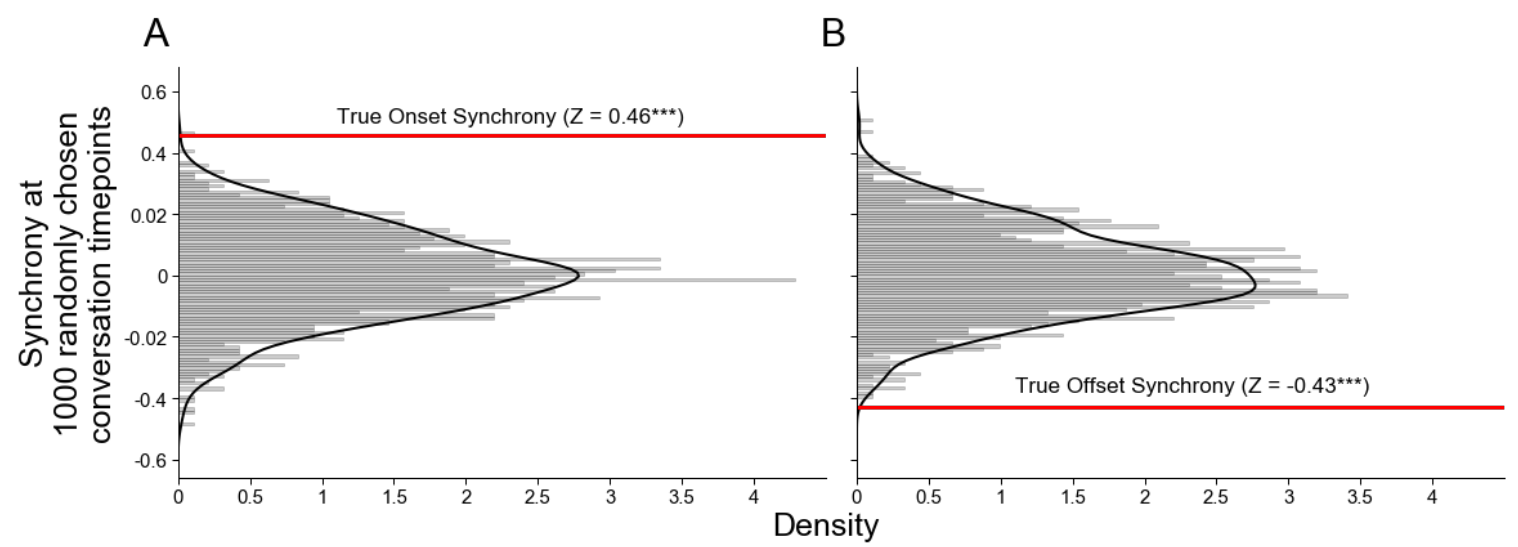

Figure 4. Results of two permutation tests comparing the A) onset and B) offset of eye contact to 265 1000 , randomly chosen, nine-second moments in each conversation, per dyad. The distributions 266 depicted above were created by taking the pupillary synchrony value at the "onset" and "offset" 
point (position 5) of each randomly chosen moment in the conversation. The true pupillary synchrony values for the onset and offset of eye contact are represented by the red horizontal lines in each figure. Pupillary synchrony at the onset of eye contact is significantly higher than would be expected by chance, and pupillary synchrony at the offset of eye contact is significantly lower than

271 would be expected by chance.

Eye contact is associated with pupillary synchrony within and between conversation turns

274 Past research has shown that eye contact often occurs as the conversation shifts from one partner 275 to the other (Kendon, 1967; Rutter et al., 1978). We therefore examined whether the effect of eye 276 contact on pupillary synchrony was similarly constrained to moments when partners passed the 277 conversational baton. We separated instances of eye-contact by whether they occurred within or 278 between conversational turns. An instance of eye contact was determined to have occurred 279 between turns if its onset began a maximum of one second prior to the start of the turn or ended a 280 maximum of one second following the end of the turn (see methods for full turn-taking 281 quantification).

283 Consistent with the prior literature, we found that within each conversation, dyads made more eye 284 contact between conversational turns $(M=63.74, S D=27.76)$ than during conversational turns ( $M$ $285=33.36, \mathrm{SD}=14.91 ; \mathrm{t}(46)=7.82, \mathrm{p}<0.001)$. Across all dyads, eye contact occurred between turns $286 \quad 64.8 \%$ of the time, and within a turn $35.2 \%$ of the time.

To test whether pupillary synchrony associated with eye contact differed by when that eye contact 289 occurred, we computed two separate pupillary synchrony timeseries: one for eye contact between 290 conversation turns, and one for eye contact within conversation turns (see figure 5 for a 291 visualization of these two timeseries). We then computed paired two-sided t-tests at each of the 
nine points along the associated pupillary synchrony timeseries. None of these t-tests were significant (t(46) ranged from $-1.8-1.52$, p's 0.07 to 0.94$)$. Together, these findings suggest that while people do tend to make eye contact as they trade turns in conversation, the relationship between eye contact and pupillary synchrony is not confined to these moments.

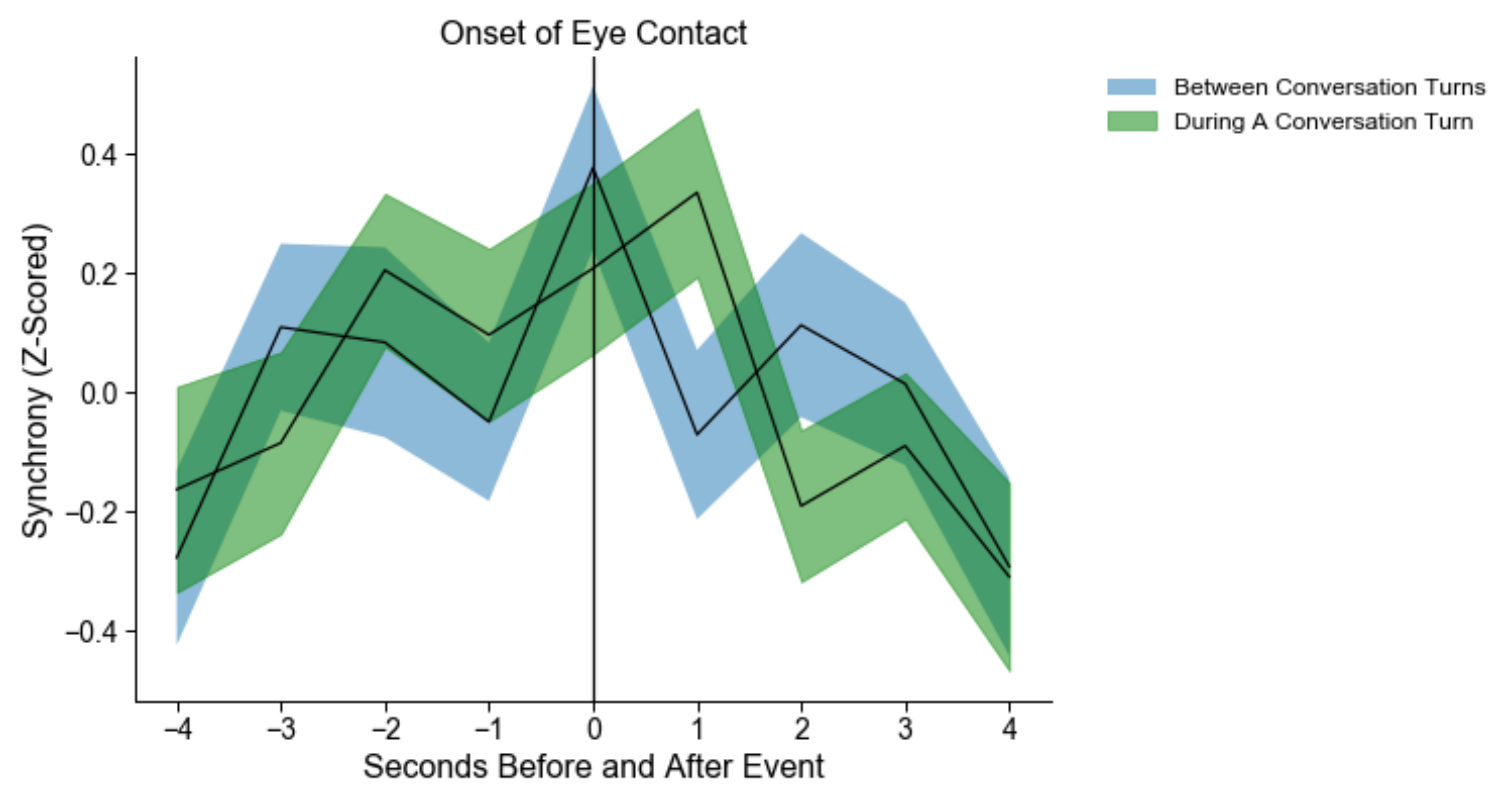

Figure 5. Visualization of pupillary synchrony at the onset of eye contact occurring within a conversation turn (standard error plotted in green) and between conversation turns (standard error plotted in blue). These two pupillary synchrony curves did not significantly differ from one another.

Given the nature of the relationship we found between pupillary synchrony and eye contact, we examined how eye contact and pupillary synchrony related to how engaged partners were by the conversation itself. Mean engagement was quantified as an average of the two continuous selfreported engagement ratings that each conversation partner made while re-watching a video of their conversation (see methods). A linear mixed effects analysis of the relationship between eye 
contact, dyads' pupillary synchrony, and their continuous mean engagement was performed in $\mathrm{R}$ using the Ime4 package (Bates et al., 2014). For this analysis, pupillary synchrony was again 310 computed in one-second windows using DTW and log-transformed to correct for homoscedasticity, 311 and dyads' time series of eye contact and mean engagement were down-sampled to $1 \mathrm{hz}$. The 312 model included fixed effects and an interaction term for pupillary synchrony and eye contact and 313 random intercepts for dyads in order to see how the interplay of eye contact and pupillary synchrony 314 related to dyads' self-reported engagement.

316 There was a significant main effect of eye contact on mean engagement $(\mathrm{t}(28160)=2.83, \beta=$ $3170.028, \mathrm{Cl}=0.01-0.05, \mathrm{p}=0.006)$, such that dyads were more engaged during eye contact than 318 in the absence of eye contact. This result was also robust to within-subjects $(p=0.002)$ and 319 between-subjects $(p=0.02)$ permutation tests, suggesting that not only are eye contact and 320 engagement significantly related to one another, but they are also related to one another in ways 321 that are unique to the individual structure of a dyad's conversation.

322 There was also a significant relationship between pupillary synchrony and mean engagement $323(\mathrm{t}(28170)=1.965, \beta=0.012, \mathrm{Cl}=0-0.02, \mathrm{p}=0.05)$, suggesting that more pupillary synchrony 324 also predicted more dyadic engagement. While this result was robust to a within-subjects 325 permutation test $(p=0.02)$, we found this result was largely driven by the fact that all dyads tended 326 to become both more synchronous and more engaged over the course of their conversations. 327 Therefore, this effect did not survive a between-subjects permutation test $(p=0.56)$.

329 Finally, we found a marginal interaction effect between eye contact and pupillary synchrony on 330 engagement $(\mathrm{t}(28150)=-1.83, \beta=-0.017, \mathrm{Cl}=-0.04-0, \mathrm{p}=0.08)$. This interaction suggests that 331 eye contact may act as a moderator for the effects of pupillary synchrony on engagement such that when dyads were not making eye contact, pupillary synchrony and engagement were positively 
related, but when dyads were making eye contact, pupillary synchrony and engagement were negatively related. This result was robust to a within-subjects permutation test $(p=0.04)$, but not to a between-subjects permutation test $(p=0.12$; see supplementary information for a more detailed description of permutation tests and visualizations of all permutation distributions).

\section{Discussion}

Here, we demonstrate that eye contact marks moments of shared attention, as measured by dyadic pupillary synchrony, in natural conversation. Further, this link is temporally directed: eye contact does not precede pupillary synchrony but rather commences as pupillary synchrony peaks and persists through its decline. This temporal relationship suggests that the onset of eye contact may not only be coincident with high shared attention (peak pupillary synchrony) but may also provide a signal of the same for conversation partners. The immediate decline in pupillary synchrony, which persists until eye contact breaks, suggests that eye contact may play a role in disrupting shared attention, perhaps to facilitate independent (non-shared) contributions. Finally, we found that dyads reported being more engaged by their conversations when they were making eye contact compared to when they were not. Together these findings support the idea that eye contact, or its neural corollaries, may facilitate social interaction by signaling moments of high attentional coupling and then aiding attentional decoupling as needed to maintain engagement.

The prevailing theory in the literature is that synchrony, referring to a wide variety of coupled behaviors, is a precursor to successful communication, shared understanding, and many other prosocial benefits (Hasson \& Frith, 2016; Launay et al., 2016; Mogan et al., 2017; Wheatley et al., 2012). Synchrony is, indeed, a robust signal of shared understanding and has been shown to causally increase feelings of rapport (Hove \& Risen, 2009; Ramseyer \& Tschacher, 2011; Wiltermuth \& Heath, 2009). These effects are strong and compelling, even showing that brain-tobrain synchrony while watching videos predicts friendship (Parkinson et al., 2018) and underscoring 
the need to study interacting humans rather than passive stimuli (Hari, et al., 2015). However, it is not clear that more interpersonal synchrony is always better, regardless of the task.

Our findings here are in line with a growing body of work suggesting that moving out of synchrony may also be an important feature of social interaction. The need to occasionally break synchrony may be especially true when individual exploration and creativity is required, as with acts of cocreation. More securely attached infants and adults tend to show less synchrony during a mirror game when interacting with their mothers and study partners, respectively, suggesting that more security in relationships might allow for more independent exploration (Beebe \& Steele, 2013; Feniger-Schaal et al., 2016). In a joint construction task, where dyads were instructed to build a model car, researchers found that synchrony was associated with more constrained construction strategies, and those dyads who worked more asynchronously (i.e., had less synchronous hand

371 movements while building) created superior model cars (Wallot et al., 2016). A study investigating 372 synchrony in a body movement task found that, while dyads who moved more synchronously 373 experienced more positive affect overall, they also had more trouble with self-regulation of that 374 affect (Galbusera et al., 2019). Finally, a study investigating interpersonal coordination during 375 conversation found that dyads decoupled their head movements during conversation in a way that 376 tracked with their status as a speaker or listener (Hale et al., 2020). Synchrony and asynchrony appear to provide differential benefits and work together to create successful interaction.

These findings are consistent with recent papers demonstrating the tendency for dyads to move continuously in and out of synchrony with one another. For example, even when participants were 381 instructed to synchronize their behavior in an improvisational joint mirroring task, researchers found 382 that interaction quality was maximized when participants sacrificed synchrony at times in favor of 383 more novel and challenging mirroring interactions (Ravreby, et al., 2021), and a model that accounted for periodically entering and exiting synchrony provided the best fit for their joint motion 
(Dahan, et al., 2016). Building on this finding, Mayo \& Gordon (2020) proposed a new model of interpersonal synchrony in which the tendencies to synchronize as well as act independently both exist during social interaction, and that the ability to move flexibly between these two states is the marker of a truly adaptive social system. Their model incorporates ideas from complex dynamical systems and treats the tendency to move flexibly in and out of synchrony as a system that is metastable. They provide evidence for their model by demonstrating that individuals who have less severe social symptoms of Autism Spectrum Disorders (ASD) tend to move in and out of eye contact with their conversation partner in a more meta-stable way. Our findings mirror and extend these findings to natural social interaction, suggesting that eye contact may index coordination between synchrony and asynchrony and thus shared and independent thought. In this way, while heightened pupillary synchrony may indicate conversational convergence, eye contact may help coordinate this convergence with independent contributions from each conversation partner.

397 Although we found no differences in how eye contact related to pupillary synchrony during conversation turns versus between conversation turns, it is possible that other coordinative mechanisms of conversation could predict pupillary synchrony (e.g. topic shifts —Egbert, 1997; 400 turn taking —Mortensen, 2011; and even blinking —Hömke, et al., 2017). In particular, Turn 401 Construction Units (TCUs; Clayman, 2013; Sacks, et al., 1978) -segments of conversation within turns that communicate complete thoughts - could also predict moments of shared attention.

Our study used unconstrained, naturalistic conversation to measure how dyads couple and decouple attention while interacting. We found that eye contact, a ubiquitous and universal feature 406 of conversation, predicts shifts in pupillary synchrony, a measure of shared attention. Specifically, 407 our results suggest that eye contact marks breakpoints in pupillary synchrony, perhaps facilitating 408 movement into and out of attentional alignment and, in so doing, facilitating engaging conversation. 409 These findings raise many questions for further research—both for typical and atypical neurological 410 populations-about how attentional states are modulated during interaction with downstream 411 consequences on how minds engage with each other. 


\section{Materials and Methods}

\section{$414 \quad$ Materials}

415 Participants

416186 subjects comprising 93 dyads (mean age: 19.38; 120 females) participated in the study.

417 Subjects were recruited from Dartmouth College, provided written informed consent approved by

418 the Committee for the Protection of Human Subjects Institutional Review Board at Dartmouth

419 College, and were compensated either monetarily or with extra course credit for participation.

420 Informed consent was obtained prior to the start of the study. After the study had concluded,

421 subjects indicated consent to the release of the videos obtained during their conversations for use

422 in scientific presentations or publications. All subjects had normal or corrected-to-normal vision.

\section{Eye-Tracking Data Collection}

425 Pupil dilation data was collected continuously while dyads engaged in conversation. Pupil diameter 426 was recorded from both eyes at $60 \mathrm{~Hz}$ using SensoMotoric Instruments (SMI) wearable eye-tracking 427 glasses (SensoMotoric Instruments, Teltow, Berlin) or at $200 \mathrm{hz}$ using Pupil Labs wearable eye428 tracking glasses (Pupil Labs, Berlin, Germany). Dyads were seated across from one another at a 429 distance of approximately 3 feet in a luminance-controlled testing room. Lux values were recorded 430 at multiple points in the room to ensure that luminance in the room did not exceed the 150 lux 431 necessary to elicit a luminance-induced pupil dilation (Maqsood, 2017). We aligned the dyads' eye432 tracking recordings in time using audio obtained from each pair of eye-tracking glasses and 433 FaceSync software (Cheong et al., 2019), which computes the offset in time between two audio 434 signals by correlating one with the other at each moment within a given window. It then returns the 435 offset as the time point that produces the highest correlation between the two signals. 
438 After their conversation, participants watched a video recording of their conversation and 439 continuously reported how engaged they were at each moment by moving a slider bar (PsychoPy 440 software, Peirce, 2007). Participants' continuous engagement ratings were recorded at $10 \mathrm{hz}$.

443 We annotated moments of eye contact during the conversation using ELAN annotation software 444 (Brugman et al., 2004). Annotations were obtained by watching videos recorded from a camera on 445 the eye-tracking glasses located at each participant's nasion. These videos thus captured the eyes 446 of the participant's conversation partner. Moments of individual eye contact were defined as 447 moments when the partner in the video looked straight at the participant's camera (the video's point 448 of view). Two independent annotations from two independent raters were obtained for each 449 participant, and only moments in which the two annotations agreed were accepted as individual 450 eye contact annotations for that participant. Each participant's individual eye contact annotations 451 were then compared with the eye contact annotations of their conversation partner to find moments 452 of mutual eye contact (when both participants were looking at each other's eyes at the same time). 453 Henceforth, mutual eye contact will be referred to simply as "eye contact," consistent with the lay 454 use of the term. An illustration of this process can be found in figure 6 .

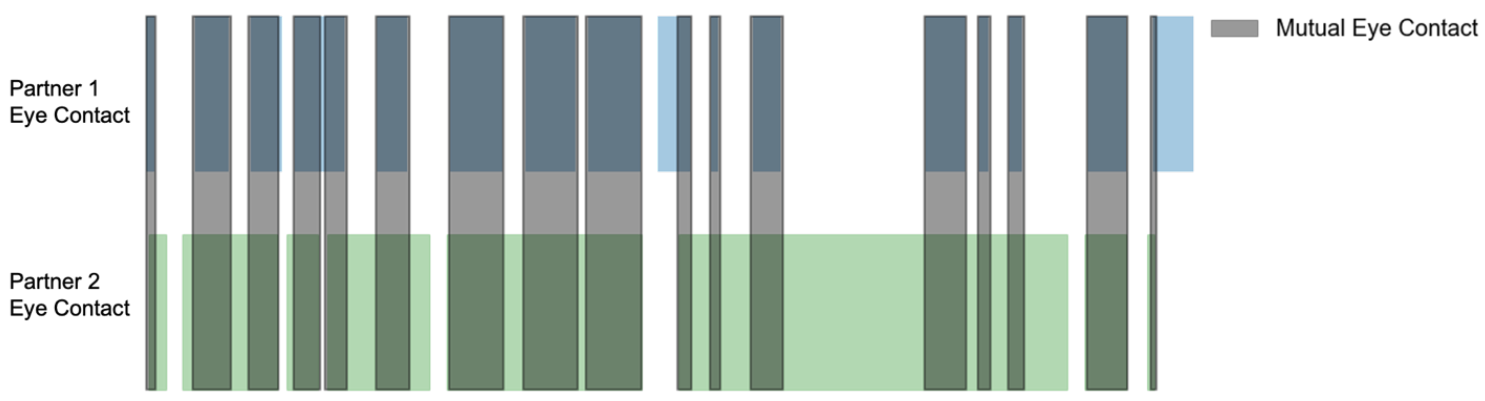


Figure 6. Annotating instances of eye contact. Individual eye contact for each conversation partner, denoted by the green and blue squares, was determined by comparing moments of individual eye

459 contact marked by each independent annotator, and accepting all moments where the annotations 460 agreed eye contact was being made. Mutual eye contact, denoted by the gray bars, was calculated 461 by comparing eye contact across conversation partners and recording moments where both partners were looking at each other's eyes.

Because of the way these moments of eye contact were obtained, there is a possibility that some 465 moments of face gaze were mistakenly recorded as moments of eye gaze, by both independent raters. Previous research has demonstrated that looking at the face area still produces the perception of eye contact (Rogers et al., 2019), suggesting that if some such moments were 468 included, they would likely achieve the same dyadic consequence as eye contact given the equivalent perception by the recipient.

470 Procedure

471 Conversation Phase

472 Dyads were seated across from one another and videotaped and eye-tracked while they engaged 473 in a ten-minute, unstructured conversation. Before their conversations, dyads received the 474 following instructions:

In this portion of the study, you will be having a conversation. Your eyes will be tracked during this conversation, and the conversation will also be video and audio recorded. Treat this conversation however you normally treat conversations in your daily life. You can talk about anything you would like. I know the glasses look a little funny, but try to ignore them during your conversation. Also, because the glasses don't work as well when you move around, try to remain still, though you 
After each conversation, a video of the conversation was saved and used to obtain participants'

487 continuous ratings of how engaged they were during the conversation. Participants were seated at 488 computers in separate rooms and instructed to watch the video of the conversation they just had, 489 while moving a computer mouse-operated slider bar to indicate how engaged they were in the 490 conversation from moment to moment. Participants received the following instructions:

You will be watching the video-taped conversation that you just had. As you watch, think back to how you were feeling during that conversation. Your task is to report how engaging you felt the conversation was. You will make these ratings by moving the computer mouse. These ratings will not be shared with your study partner. Please do your best to accurately report how engaged you felt at each point in time.

500 Participants remained seated at the computer in separate rooms and filled out a battery of 501 questions pertaining to their feelings about the conversation and their conversation partner. 502 Participants additionally completed the $A Q$ and IRI as a measure of individual differences in 503 interpersonal skills. 
505

\section{Data Analysis}

\section{Preprocessing and Quality Control}

Raw pupil dilation time series were preprocessed for further analysis by first performing linear interpolation over eye-blinks and other dropout in signal, removing any subject who required more than $25 \%$ of their data interpolated (per Kang \& Wheatley, 2017). To remove spikes, the data was then median filtered (5th order) and low-pass filtered at $10 \mathrm{~Hz}$ and finally detrended to reduce drift. Preprocessed data were then visually inspected for any remaining spikes, and a final interpolation was performed over these spikes if necessary. Data collected using Pupil Labs eye tracking glasses were down-sampled to $60 \mathrm{hz}$ to match the sampling rate of data collected using SMI eye tracking glasses.

Because of the naturalistic nature of the experiment, it proved difficult to collect dyads where both partners were below the conservative $25 \%$ interpolation threshold. Movement during the conversations and individual variation in eye tracking quality, in addition to the normal dropout due to eye blinks, caused significant data loss for many participants. For two participants, eye tracking video data was corrupt, which meant that no eye contact annotations for these participants were able to be obtained. From the original dataset, 94 subjects comprising 47 dyads (mean age: 19.78; 53 females) were included for further analysis.

\section{Computing pupillary synchrony: Dynamic Time Warping}

Dynamic Time Warping (DTW) is an algorithm used to compare signals that may be offset in time (Berndt \& Clifford, 1994). We used DTW to measure synchrony between dyads' pupils during conversation, following the method used by Kang \& Wheatley $(2015,2017)$. The DTW algorithm divides two signals into a user-defined number of segments, each representing some window of time. Then, DTW calculates the cosine similarity of these segments (though other distance metrics, 
such as Euclidean Distance, can also be used). DTW then evaluates the cosine similarity values associated with comparing each time point within the segments. The time series pair that yields the

532 smallest cosine similarity value is deemed to be the time at which the content of the two signals 533 best align, and both signals are adjusted according to that cosine similarity comparison. Each 534 adjustment incurs a penalty, or a "cost" of realignment. The sum of these penalties yields an overall 535 cost value, which represents the overall effort involved in warping one signal onto the other. Higher 536 cost values indicate greater dissimilarity between two patterns, thus "synchrony" as defined here, 537 refers to the inverse DTW cost.

Because of the way DTW is calculated, the best fit of signal 1 onto signal 2 can be found in a time540 varying way, allowing for small offsets in time to be measured and understood as synchrony. We 541 chose a 1 second sampling window for calculating DTW to allow for such offsets, while also 542 capturing a larger number of eye contact instances than the window used by previous literature 543 using DTW to calculate pupillary synchrony (3 seconds; Kang \& Wheatley, 2017).

546 To investigate the directionality of the relationship between eye contact, pupil size, and pupillary 547 synchrony, we used the R package mIVAR (Epskamp et al., 2017), which is used for the analysis 548 of multivariate time series using a network framework. This method allows for the estimation of 549 temporal, contemporaneous, and between subjects networks of relationships between time series 550 variables, where variables are "nodes" in the network, and the partial correlations between those 551 variables are "edges." The temporal network is estimated by calculating the regression coefficients 552 obtained by predicting one variable at time $t$ from another variable at time $t-1$. Once the temporal 553 network has been estimated, the contemporaneous network is calculated by taking partial 554 correlations of the residuals from the temporal network. Finally, the between subjects network is 
calculated by taking partial correlations of mean-centered predictors and adding person-means as level 2 predictors.

\section{Event-Related Analysis}

559 To examine fluctuations of synchrony around single instances of eye contact, we performed an 560 event-related analysis, treating each instance of eye contact as an event and computing averages 561 of pupillary synchrony across subjects before and during these instances. First, we extracted all 562 instances of eye contact lasting one second or longer from each dyad (M number of instances per 563 conversation $=71.6, S D=25.08)$. We restricted eye contact in this way because our pupillary 564 synchrony time series were calculated at $1 \mathrm{hz}$ (see Dynamic Time Warping section of methods for 565 detailed description of our calculation). Because of this sampling rate, for instances of eye contact 566 lasting less than one second, the onset and offset of this eye contact both occur in the same 567 pupillary synchrony window. Therefore, for these short instances of eye contact, the onset and 568 offset pupillary synchrony values are the same. Since we were separately interested in pupillary 569 synchrony at the onset and offset of eye contact, an instance of eye contact where the onset and 570 offset occur in the same sampling window of pupillary synchrony could confound true pupillary 571 synchrony levels, if pupillary synchrony at the onset of eye contact is different than pupillary 572 synchrony at the offset of eye contact.

574 We then extracted pupillary synchrony in one-second epochs for the four seconds prior to the onset 575 and offset of these eye contact instances, as well as the four seconds following the onset and offset 576 of these eye contact instances. We chose to calculate pupillary synchrony in this window after 577 plotting eye contact at a range of time series lengths and finding that no matter the length, this four578 second fluctuation around the onset and offset of eye contact remained (see supplementary 
579 information). We then z-scored each dyad's averaged pupillary synchrony during these events to

580 more clearly look at the changes surrounding each instance of eye contact.

583 To investigate whether the observed fluctuations of synchrony around eye contact could be 584 explained by turn-taking, another ubiquitous form of coordination during dyadic conversation, we 585 extracted time points during dyads' conversations where speaking switched from one partner to 586 the other. To do this, we first created transcripts of dyads' conversations using Scribie audio 587 transcription services. These transcripts contained timestamps denoting when turn switches 588 occurred. We used these timestamps to determine whether or not eye contact had occurred at a 589 turn switch. Because eye contact did not often line up exactly with the onset of a turn switch in our 590 data, and has historically been found to begin prior to the actual switch from one conversation 591 partner to the other (Kendon, 1967), we allowed any instance of eye contact occurring within one 592 second of a turn switch to be considered eye contact occurring between conversation turns.

594 Acknowledgments

595 The authors thank Oriana Gamper, Maria Goldman, Peter Kaiser, and Brody McNutt for their 596 assistance with data collection and eye contact annotations.

\section{Data Availability}

599 All pupillometry, eye contact, engagement data, and de-identified conversation transcript

600 timestamps associated with this study have been made available at

601 https://github.com/sophiewohltjen/eyeContact-in-conversation. 


\section{Code Availability}

All custom scripts associated with this study have been made available at https://github.com/sophiewohltjen/eyeContact-in-conversation.

\section{References}

1. Frith, U., \& Frith, C (2010). The social brain: Allowing humans to boldly go where no other species has been. Philosophical Transactions of The Royal Society B, 365, 165-175. https://doi.org/10.1016/j.concog.2019.102862

2. Heldner, M., \& Edlund, J. (2010). Pauses, gaps and overlaps in conversations. Journal of Phonetics, 38(4), 555-568.

3. Levinson, S. C., \& Torreira, F. (2015). Timing in turn-taking and its implications for processing models of language. Frontiers in Psychology, 6, 731.

4. Stolk, A., Verhagen, L., \& Toni, I. (2016). Conceptual Alignment: How Brains Achieve Mutual Understanding. Trends in Cognitive Sciences, 20(3), 180-191.

5. Mastroianni, A., Gilbert, D., Cooney, G., \& Wilson, T. (2021). Do conversations end when people want them to? Proceedings of the National Academy of Sciences, 18(10), 1-9. https://doi.org/10.1073/pnas.2011809118

6. Mortensen, C.D. (2011). Communication Theory. Transaction Publishers.

7. Hedge, B.J., Everitt, B.S., Frith, C.D. (1978). The role of gaze in dialogue. Acta Psychologica, 42(6), 453-475.

8. Hirvenkari, L., Ruusuvuori, J., Saarinen, V.-M., Kivioja, M., Peräkylä, A., \& Hari, R. (2013). Influence of turn-taking in a two-person conversation on the gaze of a viewer. PloS One, 8(8), e71569.

9. Kendon, A. (1967). Some functions of gaze-direction in social interaction. In Acta Psychologica (Vol. 26, pp. 22-63). https://doi.org/10.1016/0001-6918(67)90005-4 
10. Binetti, N., Harrison, C., Coutrot, A., Johnston, A., \& Mareschal, I. (2016). Pupil dilation as an index of preferred mutual gaze duration. Royal Society Open Science, 3(7), 160086.

11. Jarick, M., \& Bencic, R. (2019). Eye Contact Is a Two-Way Street: Arousal Is Elicited by the Sending and Receiving of Eye Gaze Information. Frontiers in Psychology, 10, 1262.

12. Mazur, A., Rosa, E., Faupel, M., Heller, J., Leen, R., \& Thurman, B. (1980). Physiological aspects of communication via mutual gaze. AJS; American Journal of Sociology, 86(1), $50-74$.

13. Abney, D.H., Suanda, S.H., Smith, L.B., \& Yu, C. (2020). What are the building blocks of parent-infant coordinated attention in free-flowing interaction? Infancy, 1-17. DOI: 10.1111/infa.12365

14. Conty, L., Tijus, C., Hugueville, L., Coelho, E., \& George, N. (2006). Searching for asymmetries in the detection of gaze contact versus averted gaze under different head views: a behavioural study. Spatial Vision, 19(6), 529-545.

15. Senju, A., \& Hasegawa, T. (2005). Direct gaze captures visuospatial attention. Visual Cognition, 12(1), 127-144.

16. Aston-Jones, G., Rajkowski, J., Kubiak, P., \& Alexinsky, T. (1994). Locus coeruleus neurons in monkey are selectively activated by attended cues in a vigilance task. In The Journal of Neuroscience (Vol. 14, Issue 7, pp. 4467-4480). https://doi.org/10.1523/jneurosci.14-07-04467.1994

17. Rajkowski, J. (1993). Correlations between locus coeruleus (LC) neural activity, pupil diameter and behavior in monkey support a role of LC in attention. Soc. Neurosc. , Abstract, Washington, DC, 1993. https://ci.nii.ac.jp/naid/10021384962/

18. Alnæs, D., Sneve, M. H., Espeseth, T., Endestad, T., van de Pavert, S. H. P., \& Laeng, B. (2014). Pupil size signals mental effort deployed during multiple object tracking and predicts brain activity in the dorsal attention network and the locus coeruleus. Journal of Vision, 14(4). https://doi.org/10.1167/14.4.

19. Gilzenrat, M. S., Nieuwenhuis, S., Jepma, M., \& Cohen, J. D. (2010). Pupil diameter 
tracks changes in control state predicted by the adaptive gain theory of locus coeruleus function. Cognitive, Affective \& Behavioral Neuroscience, 10(2), 252-269.

20. Joshi, S., Li, Y., Kalwani, R. M., \& Gold, J. I. (2016). Relationships between Pupil Diameter and Neuronal Activity in the Locus Coeruleus, Colliculi, and Cingulate Cortex. Neuron, 89(1), 221-234.

21. Hoeks, B. \& Levelt, W.J.M (1993). Pupillary dilation as a measure of attention: A quantitative system analysis. Behavioral Research Methods, Instruments, And Computers, 25(1), 16-26.

22. Kang, O. E., Huffer, K. E., \& Wheatley, T. P. (2014). Pupil dilation dynamics track attention to high-level information. PloS One, 9(8), e102463.

23. Kang, O., \& Banaji, M. (2020). Pupillometric decoding of high-level musical imagery. Consciousness \& Cognition, 77. https://doi.org/10.1016/j.concog.2019.102862

24. van den Brink, R. L., Murphy, P. R., \& Nieuwenhuis, S. (2016). Pupil Diameter Tracks Lapses of Attention. PloS One, 11(10), e0165274.

25. Smallwood, J., Brown, K. S., Tipper, C., Giesbrecht, B., Franklin, M. S., Mrazek, M. D., Carlson, J. M., \& Schooler, J. W. (2011). Pupillometric evidence for the decoupling of attention from perceptual input during offline thought. PloS One, 6(3), e18298.

26. Wierda, S. M., van Rijn, H., Taatgen, N. A., \& Martens, S. (2012). Pupil dilation deconvolution reveals the dynamics of attention at high temporal resolution. Proceedings of the National Academy of Sciences of the United States of America, 109(22), 84568460 .

27. Shteynberg, G (2015). Shared attention. Perspectives on Psychological Science, 10(5), 579-590.

28. Prochazkova, E., Prochazkova, L., Giffin, M.R., Scholte, H.S., De Dreu, C.K.W., \& Kret, M.E. (2018). Pupil mimicry promotes trust through the theory of mind network. Proceedings of the National Academy of Sciences, 115(31), E7265-E7274.

29. Van Breen, J.A., De Dreu, C.K.W., \& Kret, M.E. (2018). Pupil to pupil: The effect of a partner's pupil size on (dis)honest behavior. Journal of Experimental Social Psychology, 
74, 231-245.

30. Kang, O., \& Wheatley, T. (2015). Pupil dilation patterns reflect the contents of consciousness. Consciousness and Cognition, 35, 128-135.

31. Kang, O., \& Wheatley, T. (2017). Pupil dilation patterns spontaneously synchronize across individuals during shared attention. Journal of Experimental Psychology. General, 146(4), 569-576.

32. Hasson, U., \& Frith, C. D. (2016). Mirroring and beyond: coupled dynamics as a generalized framework for modelling social interactions. Philosophical Transactions of the Royal Society of London. Series B, Biological Sciences, 371(1693). https://doi.org/10.1098/rstb.2015.0366

33. Leong, V., Byrne, E., Clackson, K., Georgieva, S., Lam, S., \& Wass, S. (2017). Speaker gaze increases information coupling between infant and adult brains. Proceedings of the National Academy of Sciences of the United States of America, 114(50), 13290-13295.

34. Dikker, S., Wan, L., Davidesco, I., Kaggen, L., Oostrik, M., McClintock, J., Rowland, J., Michalareas, G., Van Bavel, J. J., Ding, M., \& Poeppel, D. (2017). Brain-to-Brain Synchrony Tracks Real-World Dynamic Group Interactions in the Classroom. Current Biology: CB, 27(9), 1375-1380.

35. Kinreich, S., Djalovski, A., Kraus, L., Louzoun, Y., \& Feldman, R. (2017). Brain-to-Brain Synchrony during Naturalistic Social Interactions. Scientific Reports, 7(1), 17060.

36. Konvalinka, I., Xygalatas, D., Bulbulia, J., Schjødt, U., Jegindø, E.-M., Wallot, S., Van Orden, G., \& Roepstorff, A. (2011). Synchronized arousal between performers and related spectators in a fire-walking ritual. Proceedings of the National Academy of Sciences of the United States of America, 108(20), 8514-8519.

37. Hirsch, J., Zhang, X., Noah, J. A., \& Ono, Y. (2017). Frontal temporal and parietal systems synchronize within and across brains during live eye-to-eye contact. Neurolmage, 157, 314-330.

38. Kelley, M. S., Noah, J. A., Zhang, X., Scassellati, B., \& Hirsch, J. (2020). Comparison of human social brain activity during eye-contact with another human and a humanoid robot. Frontiers in Robotics and Al, 7. https://www.ncbi.nlm.nih.gov/pmc/articles/pmc7879449/ 39. Koike, T., Tanabe, H. C., Okazaki, S., Nakagawa, E., Sasaki, A. T., Shimada, K., 
Sugawara, S. K., Takahashi, H. K., Yoshihara, K., Bosch-Bayard, J., \& Sadato, N. (2016). Neural substrates of shared attention as social memory: A hyperscanning functional magnetic resonance imaging study. Neurolmage, 125, 401-412.

40. Koike, T., Sumiya, M., Nakagawa, E., Okazaki, S., \& Sadato, N. (2019). What Makes Eye Contact Special? Neural Substrates of On-Line Mutual Eye-Gaze: A Hyperscanning fMRI Study. eNeuro, 6(1). https://doi.org/10.1523/ENEURO.0284-18.2019

41. Noah, J. A., Zhang, X., Dravida, S., Ono, Y., Naples, A., McPartland, J. C., \& Hirsch, J. (2020). Real-Time Eye-to-Eye Contact Is Associated With Cross-Brain Neural Coupling in Angular Gyrus. Frontiers in Human Neuroscience, 14, 19.

42. Silbert, L. J., Honey, C. J., Simony, E., Poeppel, D., \& Hasson, U. (2014). Coupled neural systems underlie the production and comprehension of naturalistic narrative speech. In Proceedings of the National Academy of Sciences (Vol. 111, Issue 43, pp. E4687E4696). https://doi.org/10.1073/pnas.1323812111

43. Stephens, G. J., Silbert, L. J., \& Hasson, U. (2010). Speaker-listener neural coupling underlies successful communication. Proceedings of the National Academy of Sciences of the United States of America, 107(32), 14425-14430.

44. Chen, J., Leong, Y. C., Honey, C. J., Yong, C. H., Norman, K. A., \& Hasson, U. (2017). Shared memories reveal shared structure in neural activity across individuals. Nature Neuroscience, 20(1), 115-125.

45. Bögels, S., Magyari, L., \& Levinson, S. C. (2015). Neural signatures of response planning occur midway through an incoming question in conversation. In Scientific Reports (Vol. 5, Issue 1). https://doi.org/10.1038/srep12881

46. Redcay, E., \& Schilbach, L. (2019). Using second-person neuroscience to elucidate the mechanisms of social interaction. Nature Reviews. Neuroscience, 20(8), 495-505.

47. Schilbach, L., Timmermans, B., Reddy, V., Costall, A., Bente, G., Schlicht, T., \& Vogeley, K. (2013). Toward a second-person neuroscience. The Behavioral and Brain Sciences, 36(4), 393-414.

48. Bates, D., Mächler, M., Bolker, B., \& Walker, S. (2014). Fitting Linear Mixed-Effects 
49. Rutter, D. R., Stephenson, G. M., Ayling, K., \& White, P. A. (1978). The timing of Looks in dyadic conversation. The British Journal of Social and Clinical Psychology, 17(1), 17-21.

50. Launay, J., Tarr, B., \& Dunbar, R. I. M. (2016). Synchrony as an adaptive mechanism for large-scale human social bonding. Ethology: Formerly Zeitschrift Fur Tierpsychologie, 122(10), 779-789.

51. Mogan, R., Fischer, R., \& Bulbulia, J. A. (2017). To be in synchrony or not? A metaanalysis of synchrony's effects on behavior, perception, cognition and affect. Journal of Experimental Social Psychology, 72, 13-20.

52. Wheatley, T., Kang, O., Parkinson, C., \& Looser, C. E. (2012). From mind perception to mental connection: Synchrony as a mechanism for social understanding. Social and Personality Psychology Compass, 6(8), 589-606.

53. Hove, M. J., \& Risen, J. L. (2009). It's All in the Timing: Interpersonal Synchrony Increases Affiliation. Social Cognition, 27(6), 949-960.

54. Ramseyer, F., \& Tschacher, W. (2011). Nonverbal synchrony in psychotherapy: coordinated body movement reflects relationship quality and outcome. Journal of Consulting and Clinical Psychology, 79(3), 284-295.

55. Wiltermuth, S. S., \& Heath, C. (2009). Synchrony and cooperation. Psychological Science, 20(1), 1-5.

56. Parkinson, C., Kleinbaum, A.M., \& Wheatley, T. (2018). Similar neural responses predict friendship. Nature Communications, 9(1), 332.

57. Hari, R., Henriksson, L., Malinen, S., \& Parkkonen, L. (2015). Centrality of social interaction in human brain function. Neuron, 88, 181-193.

58. Beebe, B., \& Steele, M. (2013). How does microanalysis of mother-infant communication inform maternal sensitivity and infant attachment? In Attachment \& Human Development (Vol. 15, Issues 5-6, pp. 583-602). https://doi.org/10.1080/14616734.2013.841050 
59. Feniger-Schaal, R., Noy, L., Hart, Y., Koren-Karie, N., Mayo, A. E., \& Alon, U. (2016). Would you like to play together? Adults' attachment and the mirror game. Attachment \& Human Development, 18(1), 33-45.

60. Wallot, S., Mitkidis, P., McGraw, J. J., \& Roepstorff, A. (2016). Beyond Synchrony: Joint Action in a Complex Production Task Reveals Beneficial Effects of Decreased Interpersonal Synchrony. PloS One, 11(12), e0168306.

61. Galbusera, L., Finn, M. T. M., Tschacher, W., \& Kyselo, M. (2019). Interpersonal synchrony feels good but impedes self-regulation of affect. Scientific Reports, 9(1), 14691.

62. Hale, J., Ward, J. A., Buccheri, F., Oliver, D., \& Hamilton, A. (2020). Are you on my wavelength? Interpersonal coordination in naturalistic conversations. Journal of Nonverbal Behavior, 44, 63-83. https://doi.org/10.31234/osf.io/5r4mj

63. Ravreby, I., Shilat, Y., \& Yeshurun, Y. Reducing synchronization to increase interest improves interpersonal liking. bioRxiv [preprint] (2021). https://www.biorxiv.org/content/10.1101/2021.06.30.450608v1.full.pdf (accessed 7 July 2021).

64. Dahan, A., Noy, L., Hart, Y., Mayo, A., \& Alon, U. (2016). Exit from Synchrony in Joint Improvised Motion. PloS One, 11(10), e0160747.

65. Mayo, O., \& Gordon, I. (2020). In and out of synchrony-Behavioral and physiological dynamics of dyadic interpersonal coordination. Psychophysiology, 57(6), e13574.

66. Egbert, M.M. (1997). Schisming: The transformation from a single conversation to multiple conversations. Research on Language And Social Interaction, 30(1), 1-51.

67. Hömke, P., Holler, J., \& Levinson, S.C. (2017). Eye blinking as addressee feedback in face to face conversation. Research on Language And Social Interaction, 50(1), 54-70.

68. Clayman, S.E., (2013). Turn-constructional units and the transition-relevance place. in The Handbook of Conversation Analysis, 151-166.

69. Sacks, H., Schegloff, E.A., \& Jefferson, G. (1978). A simplest systematics for the 
organization of turn taking for conversation. in Studies in The Organization of Conversational Interaction, 7-55.

70. Maqsood, F. (2017). Effects of varying light conditions and refractive error on pupil size. Cogent Medicine, 4(1). https://doi.org/10.1080/2331205x.2017.1338824

868

71. Cheong, J. H., Brooks, S., \& Chang, L. J. (2019). FaceSync: Open source framework for recording facial expressions with head-mounted cameras. F1000 Research, 8, 702.

72. Peirce, J. W. (2007). PsychoPy—Psychophysics software in Python. Journal of Neuroscience Methods, 162(1-2), 8-13.

73. Brugman, H., Russel, A., \& Nijmegen, X. (2004). Annotating Multi-media/Multi-modal Resources with ELAN. LREC.

877

878

74. Rogers, S. L., Guidetti, O., Speelman, C. P., Longmuir, M., \& Phillips, R. (2019). Contact 880 Is in the Eye of the Beholder: The Eye Contact Illusion. Perception, 48(3), 248-252.

883

75. Berndt, D. J., \& Clifford, J. (1994). Using dynamic time warping to find patterns in time series. KDD Workshop, 10, 359-370.

76. Epskamp, S., Deserno, M. K., \& Bringmann, L. F. (2017). mIVAR: multi-level vector autoregression. $R$ Package Version 0. 4. 